\title{
The Double Diffraction Model for LEED-Intensity Spectra of the Clean $\operatorname{Pt}(100)$ Surface
}

\author{
K. Heinz, P. Heilmann, and K. Müller* \\ Institut für Angewandte Physik, Universität Erlangen-Nürnberg \\ (Z. Naturforsch. 32 a, 28-32 [1977]; received December 2, 1976)
}

\begin{abstract}
The intensity spectra of the superstructure diffraction spots of the clean platinum (100) surface are presented for normal or nearly normal incidence. Their gross features are interpreted in terms of multiple diffraction contributions. It is shown that most of the maxima in the spectra can be explained by double diffraction processes from an idealized pure hexagonal surface layer and quadratic bulk layers. Alternative models in which the surface layer exchanges already all occuring reciprocal lattice vectors would predict additional peaks which, however, do not arise. Therefore superstructure features of the surface layer are assumed to be of second order. Thus double diffraction involving only idealized hexagonal and quadratic structures seems to dominate the diffraction behaviour of clean $\mathrm{Pt}(100)$.
\end{abstract}

\section{Introduction}

The complicated LEED-pattern of platinum (100) has been investigated in a series of papers ${ }^{1-5}$, in some cases however only as an analogy of the very similar (100)-surface of gold. There is no doubt that the superstructure results from reorganization of the top layer from a quadratic structure to hexagonal close packing. However, the actual physical process responsible for the pattern is still uncertain. The observed extra spots of the superstructure unit mesh are either due to multiple (double) diffraction between a more or less pure hexagonal first layer and the bulk, or to kinematic diffraction by a puckering of this layer, or both.

Further insight is generally expected from full dynamical calculation of diffraction intensities and their comparison with experiment. However, at the present stage of theory, the large size of the superstructure unit mesh requires a vast amount of computational efforts. But a development of the full dynamical theory in terms of multiple diffraction contributions ${ }^{6}$ can already be useful in some cases. This approach seems to be applicable to platinum (100) for normal incidence of the primary beam as it was demonstrated in an earlier paper ${ }^{7}$. If first and second order diffraction processes are the main contributions to the diffraction pattern of $\mathrm{Pt}(100)$ it should be interesting to analyse particularly the spectra of the extra beams. Therefore the intensity

Reprint requests to Prof. Dr. Klaus Müller, Institut für Angewandte Physik, Lehrstuhl für Festkörperphysik, Universität Erlangen-Nürnberg, Erwin-Rommel-Straße 1, D.8520 Erlangen, West-Germany. spectra of these extra beams are presented in this paper and are discussed with respect to their gross structure.

\section{Double Diffraction Model of the $\operatorname{Pt}(100)$ Superstructure}

Early considerations made for the similar (100) surface of gold excluded the multiple or - more simple - double diffraction model on the basis of two objections ${ }^{1}$. First it was stated that not all observed extra spots could be predicted. This was a consequence of involving only the lowest order of reciprocal lattice vectors in double diffraction processes. It will be demonstrated below that all observed extra spots can be predicted if larger reciprocal lattice vectors are implied. The second objection came from some extra spots which still exist at low energies, where one of the beams from which they originate by double diffraction is evanescent. However, as it was pointed out by Palmberg and Rhodin $^{3}$, this is possible, because at low energies the electron wave length is more than twice the layer distance and so evanescent waves cannot be neglected.

Figure 1 a shows the LEED pattern of the clean $\mathrm{Pt}(100)$ surface. No impurities were detected at this stage in the retarding field Auger spectrum. The pattern was taken at nearly normal incidence at $E=$ $60 \mathrm{eV}$ and shows all known spots along the [0T] direction where the crystal holder does not mask the pattern. The triplet at $0 \frac{\overline{4}}{5}$ cannot be seen at the chosen energy. The geometry of the LEED pattern including all spots appearing at various energies 
leads to a size of the supercell of $(5 \times 25)$ which deviates slightly from the value of $(5 \times 20)$ accepted so far by most authors in literature. However, as the coincidence in [01] direction seems to be more accidental than systematic the term $(5 \times 25)$, or $(5 \times 20)$ superstructure is misleading which was already pointed out in other papers ${ }^{8,9}$. The pattern of one unit mesh and for one of two orthogonal domains is repeated in Fig. $1 \mathrm{~b}$, where spots of the quadratic substrate are indicated by square symbols, those of the hexagonal surface by triangles. The remaining spots can be easily constructed by linear combination of the corresponding bulk and surface reciprocal lattice vectors ( $g$ and $\hat{g}$ respectively). So the spots represented by large full circles are generated by reciprocal lattice vectors within the primitive unit meshes, i. e. $|g|^{2}+|\hat{g}|^{2}=G^{2} \leqq|01|^{2}+$ $\left.\hat{01}\right|^{2}$. An example is $0 \frac{1}{5}=\hat{01}+0 \overline{1}$, considering that $\hat{01}$ is equivalent to $0 \frac{6}{5}$. Additional spots occur, indicated by crosses, when the limit for $|G|$ is increased to $G^{2} \leqq 11^{2}+\hat{01}{ }^{2}$. Correspondingly open circles belong to $G^{2} \leqq 20^{2}+\hat{01^{2}}$ and small full circles to $G^{2} \leqq 21^{2}+0 \hat{\mathrm{l}}^{2}$. This choice for $|G|$ already represents all observed spots. With further increasing length of the exchanged reciprocal lattice vectors more spots would become possible which, however, do not appear. So the LEED pattern can be built up by low order reciprocal vectors, and higher order vectors can be neglected. In this general way the double diffraction model is simple and successful. In Figure $1 \mathrm{c}$ it is supported by optical simulation, where the diffracted beams of a hexagonal grating were sent through a second grating with quadratic structure. This method was described by Hilferink et al. ${ }^{10,}{ }^{11}$, the pattern in Fig. $1 \mathrm{c}$ was produced in the same manner.

On the other hand one consideres a puckering of the top layer due to the misfit with the substrate. Such a surface puckering model would - in principle - predict the whole assembly of all $(5 \times 25)$ spots from the simple kinematic point of view. However special formation of the puckering lowers the intensity of absent spots down to an undetectable amount ${ }^{1,4,5}$. This apparent correspondence to the double diffraction picture results, if the interaction between surface and bulk leading to puckering contains only those reciprocal lattice vectors of the undisturbed surface and substrate with considerable weight, which are involved in the "pure double dif-
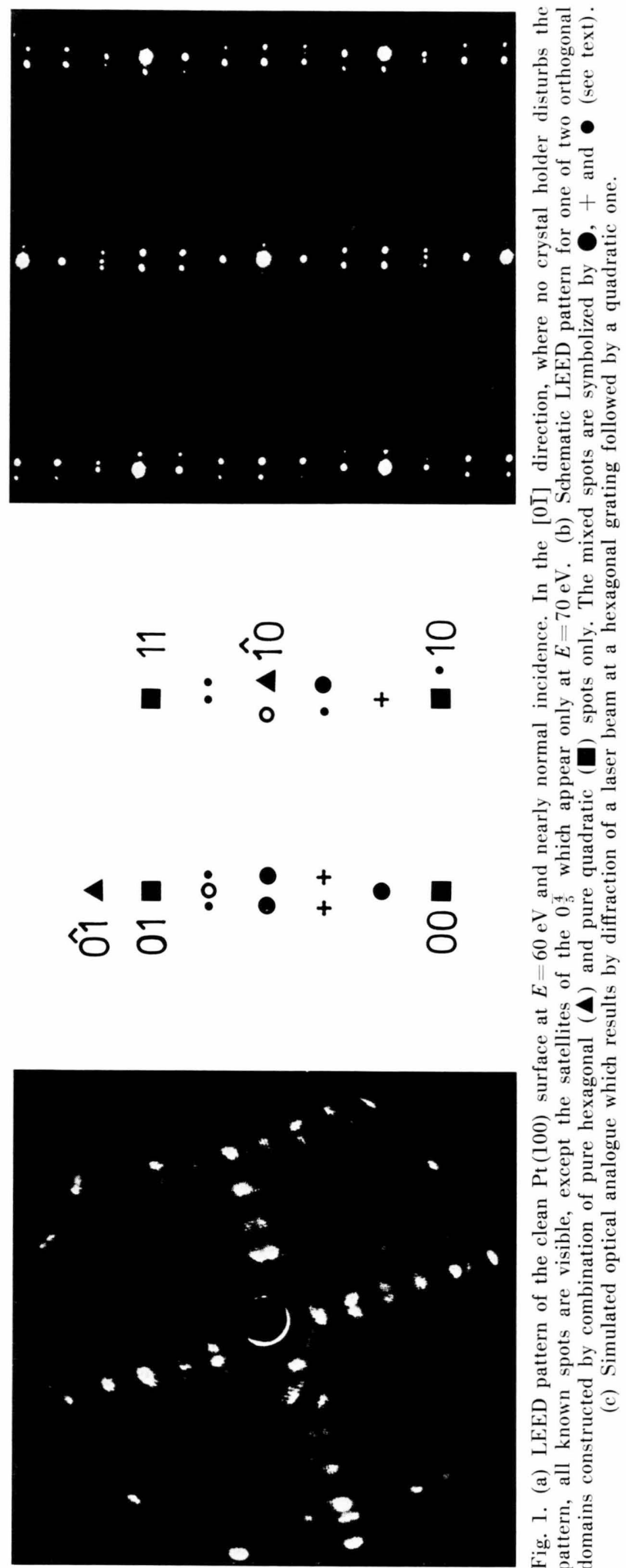
fraction" model. So the appearance or nonexistance of spots alone does not distinguish between the multiple diffraction and the puckering approach. Therefore only intensities can favour one of the models to be more realistic than the other.

\section{Intensity Spectra and Interpretation}

The normalized spectra $I(E) / I_{0}(E)$ of the extra beams along one axis of the unit mesh are given in Figure 2. They show more structure than those
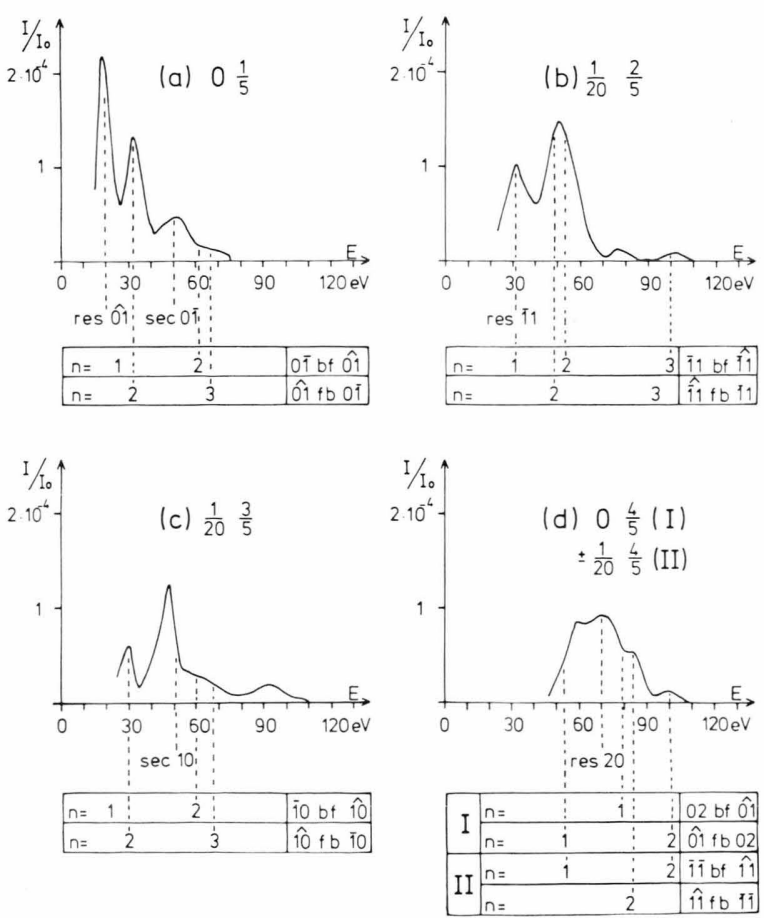

Fig. 2. LEED intensity structures for normal incidence, except the $0 \frac{1}{5}$ spot which is taken at $\Theta=2.5^{\circ}$. Tables below the spectra give the positions due to double diffraction interference maxima. The lines stand for the different processes, indicated on the right, the numbers in the lines give the order of interference.

published earlier ${ }^{5}$, possibly due to a new procedure of measurement which allows careful background subtraction ${ }^{12}$. All beams are measured for normal incidence except $0 \frac{1}{5}$ which was taken at $\Theta=2.5^{\circ}$. The normal incidence allowed to measure doublets as a whole and to divide the intensities afterwards by two for symmetry arguments. The three beams forming the triplet at $0 \frac{4}{5}$ had to be measured also as a whole because of overlap at some energies.
Since they are not equivalent, separation after measurement was not possible in this case.

Similar intensity spectra have been used for a Patterson function evaluation ${ }^{5}$. Such an approach, however, does not seem appropriate for at least two reasons. At first, the success of this procedure in X-ray diffraction is due to single scattering only. There is no profound knowledge if or how it can be modified to unaveraged LEED-spectra where multiple (double) scattering is undoubtedly important. Secondly, as LEED data are limited on both the high and low energy side of the spectra, truncation affects the Patterson function and leads possibly to misinterpretation ${ }^{13}$. On the other hand a kinematic calculation starting with a reasonable puckering model ${ }^{4}$ shows no satisfactory agreement with the measured intensities.

Of course only calculations using the consistent dynamical theory could interprete the measured intensities in detail. However dynamical contributions can also be considered by a development in terms of multiple diffraction orders given by Mc Rae $^{6}$. This method is applicable to cases of rapid decrease of higher order diffraction contributions. For normal incidence of the primary beam it allows to attribute observed peaks in the intensity spectra to physical diffraction processes. This was successfully demonstrated for the specular beam of a $\mathrm{Pt}$ (100) pattern in an earlier paper ${ }^{7}$. The method is based on the assumption that the diffraction behaviour of a single atomic layer varies smoothly with energy. This is true for layers where the atoms are arranged within a plane in contrast to a heavily puckered platinum top layer. However, if we assume that the puckering of the first layer is required by a constant metallic bond length of $2.77 \AA$ between neighboured platinum atoms, a puckering amplitude of $0.4 \AA$ results. Interference effects within the layer itself are therefore expected only for $\lambda / 2 \leqq 0.4 \AA$ which is beyond the relevant energy range in the spectra. So a smooth diffraction behaviour of the surface layer for $E \leqq 100 \mathrm{eV}$ is probable. Moreover, as puckering dies away rapidly with increasing penetration into the crystal, it might be reasonable to assume only weak kinematic contributions from this modulation in multilayer interference. The structure of the spectra is therefore probably dominated by multiple scattering between surface and bulk rather than by puckering modulation. Consequently we shall perform the same analysis of 
the spectra as in the paper mentioned above ${ }^{7}$ and use the inner potential evaluated there for peak position correction.

First we have to determine the momentum which can be exchanged by the different layers. While volume layers can exchange only vectors of the quadratic reciprocal lattice, two possibilities exist for the surface layer. The first and simpler one allows only exchanges due to the hexagonal close packing, the second assumes that all reciprocal lattice vectors occuring in the LEED pattern are involved. In the first case the $0 \frac{1}{5} \mathrm{spot}$, for instance, would result from the processes $0 \frac{1}{5}=0 \overline{\mathrm{I}}+\hat{0 \mathrm{l}}$ or $0 \frac{1}{5}=\hat{01}+0 \overline{\mathrm{I}}$ which will be abbreviated in the following by the terms $(0 \overline{\mathrm{I}}$ bf $0 \hat{\mathrm{I}})$ and $(\hat{\mathrm{O}} \mathrm{fb} 0 \overline{\mathrm{I}})$ indicating for the first process that $0 \overline{\mathrm{I}}$ is exchanged in backward direction at a bulk layer and then 01 in forward direction at the surface layer. In the second case, $0 \frac{1}{5}$ is exchanged at once at the puckered surface and volume layers contribute only by specular reflection. However the peak positions corresponding to this simple process do not agree with the observed structure. By the way, this is also true for predicted kinematic maxima which result when puckering is assumed for bulk layers too.

So there is some evidence that the structure of intensity spectra is dominated by double diffraction processes of the first kind described. Again considering the $0 \frac{1}{5}$ spot, maxima are expected to arise at first order Bragg positions of the $0 \overline{\mathrm{I}}$ beam (at normal incidence for the bf process and at oblique incidence for the $\mathrm{fb}$ process). The maxima positions are given in the table below the spectrum in Figure $2 \mathrm{a}$. The different lines of the table correspond to the different processes while numbers in the lines give the order $\mathrm{n}$ of interference. For the $0 \frac{1}{5}$ spectrum only the second peak seems to correspond clearly to a diffraction process $\mathrm{fb}$, perhaps also the feature at about $65 \mathrm{eV}$. The process bf seems to influence the spectrum not at all. This is consistent with the behaviour of the $0 \bar{I}$ spectrum, which was also measured but is not presented here. It shows only very low intensity in the relevant energy range apart from a heavy peak at $52 \mathrm{eV}$, which was interpreted as a second order Bragg peak. It can be associated with the third peak in Figure 2 a. This correspondance demonstrates the possible importance of higher order contributions (an equivalent contribution occurs in the spectrum of the $\frac{1}{25} \frac{3}{5}$ spot in Figure 2 c). The remaining first peak in Fig. 2 a can be interpreted by the fact that increasing scattering in this energy range is strongly diminished by the disappearance of the $\hat{01}$ beam which becomes evanescent inside the crystal when the energy is decreased below $20 \mathrm{eV}$. As this energy corresponds to a $\hat{01}$ resonant beam running within the surface layer itself, this peak is labelled res $\hat{0 l}$.

In the same way the other spectra in Fig. 2 can be interpreted, however with less significance for the $0 \frac{4}{5}$ triplet because of the three beams involved. The overall result is, that in most cases peaks in the spectra can be associated with double diffraction processes. Some peaks, however, require contributions from higher order multiple diffraction. In a few cases maxima cannot be identified (e.g. the peak at $92 \mathrm{eV}$ in Figure $2 \mathrm{c}$ ).

We should point out that third order contributions in our "pure model" (pure hexagonal and pure quadratic structures) cannot be distinguished in the structure of intensities from second order contributions in the "mixed model", where the surface layer momentum exchange already leads to all occuring beams. However, as mentioned above, mixed contributions from the surface layer and specular reflections at the following layers do not occur with appreciable weight. So we can at least say that the structure of non integer spot intensities is dominated by double diffraction processes originating from momentum exchange with the surface layer of hexagonal symmetry and with the bulk layers of quadratic symmetry.

\section{Conclusion}

Most of the peaks in the spectra of superstructure spots in the diffraction pattern of clean $\mathrm{Pt}(100)$ can be interpreted by double diffraction processes exchanging reciprocal vectors of a pure hexagonal structure at the surface and of the undisturbed bulk structure. Peaks, which remain unidentified within this approach, are assumed to be due to higher order than double diffraction processes. Some third order processes in the "pure model" canot be distinguished from double diffraction processes in a modified model, which assumes a surface layer that exchanges all reciprocal vectors occuring in the diffraction pattern. However the weight of such processes seems to be negligible: Already the simplest of them, which collect the total momentum exchange at the modified surface layer followed by only 
specular reflection at successive layers, do not appear to contribute to the intensity spectra at the expected energy. Therefore, the assumption that diffraction at the $\operatorname{Pt}(100)$ surface is dominated by double diffraction processes involving pure hexagonal and quadratic structures is reasonable. This is equivalent to a model, where the influence of the quadratic sublayer on the hexagonal surface layer is only of second order compared with its pure hexagonal structure. Therefore the described double dif-

${ }^{1}$ D. G. Fedak and N. A. Gjostein, Surface Sci. 8, 77 [1967].

2 A. E. Morgan and G. A. Somorjai, Surface Sci. 12, 405 [1968].

3 P. W. Palmberg and T. N. Rhodin, J. Chem. Phys. 49, 134 [1968] ; 147 [1968].

4 W. D. Robertson, J. Vacuum Sci. Technol. 8, 403 [1971].

5 T. A. Clarke, R. Mason, and M. Tescarí, Surface Sci. 40, 1 [1973].

${ }^{6}$ E. G. McRae, Surface Sci. 11, 479 [1968]; 492 [1968].

7 K. Heinz, N. Lieske, and K. Müller, Z. Naturforsch. 31 a. $1520[1976]$. fraction model essentially touches the main features of the $\mathrm{Pt}$ (100) surface.

\section{Acknowledgement}

This work was supported by Deutsche Forschungsgemeinschaft (DFG). The authors wish to thank N. Lieske for making available part of his intensity measurements and W. Paulini for testing the double diffraction model by optical simulation.

8 F. Grønlund and P. E. Højlund Nielsen, J. Appl. Phys. 43, 3919 [1972].

9 K. Müller, P. Heilmann, K. Heinz, and G. G. Waldecker, Vak. Technik 25, 227 [1976].

${ }^{10}$ H. Hilferink, C. Klingshirn, and K. Müller, Z. Naturforsch. 29 a, 1512 [1974].

11 K. Heinz, Z. Naturforsch. 29 a, 1514 [1974].

12 P. Heilmann, E. Lang, K. Heinz, and K. Müller, Appl. Phys. 9, 247 [1976].

13 D. P. Woodruff. K. A. R. Mitchell, and L. McDonell, Surface Sci. 42, 355 [1974]. 